\title{
The Relation between the Ejection Mechanism and Ion Abundance in the Electric Double Layer of Droplets
}

\author{
Victor Kwan, ${ }^{\dagger}$ Ryan O’Dwyer, ${ }^{\dagger}$ David Laur, ${ }^{\dagger}$ Jiahua Tan, ${ }^{\dagger},{ }^{\ddagger}$ and Styliani \\ Consta*,† \\ $\dagger$ Department of Chemistry, The University of Western Ontario, London, Ontario, Canada \\ N6A $5 B 7$ \\ $\ddagger$ College of Chemistry, Nankai University, Tianjin, P. R. China 300071 \\ E-mail: sconstas@uwo.ca
}

\section{Abstract}

Charged droplets have been associated with distinct chemical reactivity. It is assumed that the composition of the surface layer plays a critical role in enhancing the reaction rates in the droplets relative to their bulk analogues. We use atomistic modeling to relate the localization of the ions in the surface layer to their ejection propensity. We find that the ion ejection takes place via a two-stage process. Firstly, a conical protrusion emerges as a result of a global droplet deformation that is insensitive to the locations of single ions. The ions are subsequently ejected as they enter the conical regions. The study provides mechanistic insight into the ionevaporation mechanism, which can be used to revise the commonly used ion-evaporation models. We argue that atomistic molecular dynamics simulations of minute nano-drops, do not sufficiently distinguish the ion-evaporation mechanism from a Rayleigh fission. We explain mass spectrometry data on the charge state of small globular proteins and the existence of super-charged droplet states that have been detected in experiments.

\section{Introduction}

Multiple charged droplets are found in a number of highly diverse environments such as thunderclouds ${ }^{1}$ ink-jet printing, ${ }^{2}$ mass spectrometry ionization methods, ${ }^{3-11}$ and in the emerging area of aerosol micro-reactors. ${ }^{12-19}$ The relation of the structure of a multiple charged droplet to the ion-ejection mechanisms has been a central question in mass spectrometry and atmospheric chemistry. The problem is significant because the (simple) ion-ejection may determine the charge state of macroions that are detected in native mass spectrometry. ${ }^{20-22}$ In the last half-century several models of ionejection have been developed. ${ }^{23-26}$ The models have been empirical as they have been lacking atomistic level information about the structure of a charged droplet.

In prior publications we have reported on the droplet composition and electric properties using atomistic modeling. ${ }^{27-30}$ In this article, we relate the equilibrium droplet structure to the preferential ion ejection when a mixture of ions is present. Our study provides detailed mechanistic insight that is not captured in the ionevaporation models. In Fig. 1 we classify the variety of processes that take place in charged droplets. In the first column we show the three scenarios of droplet breakdown that have been 
hypothesized. In the second column we show the higher complexity that the presence of a macroion induces.
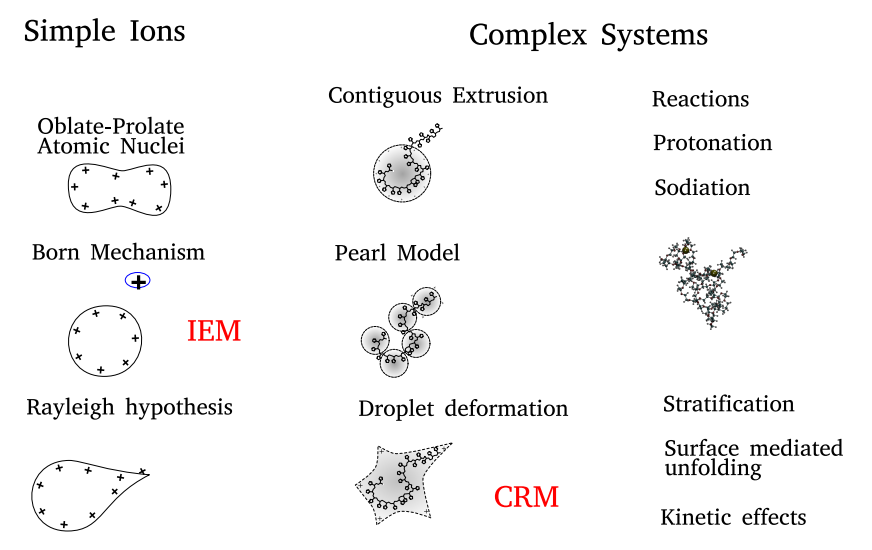

Figure 1: Classification of the (macro)ion-drop interactions. CRM stands of charged-residue model $^{20,31}$ and IEM for the ion-evaporation mechanism. ${ }^{23-25}$ The first column shows the scenarios for droplet disintegration, when a droplet comprises solvent and single ions. The second column shows how a macroion affects the droplet morphologies and ion transfer reactions that take place. ${ }^{32-35}$ The term "stratification" indicates the layered solvent structure that may be induced in a droplet by the charge of a macroion. Example of stratification appears in a $\mathrm{H}_{2} \mathrm{O}-\mathrm{CH}_{3} \mathrm{CN}$ mixture of solvents. ${ }^{36}$ Surface mediated unfolding refers to the change in the conformation of a macromolecule when it is transferred from the bulk solution to the gaseous phase. Typical example is poly(ethylene glycol), which is compact in an aqueous bulk solution and unfolds in a droplet. ${ }^{37}$

A droplet disintegrates via solvent evaporation and ion ejection (for ion ejection see first column in Fig. 1). Ion ejection may take place either via a Rayleigh mechanism ${ }^{38}$ or ion evaporation $^{23,24}$ (IEM). The Rayleigh mechanism involves the release of a substantial amount of charge from jets formed on the droplet. IEM has been defined as the release of a single solvated ion from the parent drop before the Rayleigh limit is reached. Macroscopic models of the ion-evaporation mechanism have been developed by Iribarne-Thomson ${ }^{23,24}$ and
Labowsky-Fenn-de la Mora. ${ }^{25,39}$ Both models consider a charged spherical droplet at equilibrium and they treat the release of a single ion by first order kinetics. In the kinetic equation the total concentration of the ions in the droplet is considered. The models treat differently the activation energy barrier but in both, the backbone of the analysis includes droplet's surface energy and Born solvation energy of the ions. It has been supported experimentally that IEM occurs in droplets with a radius less than $10 \mathrm{~nm} .{ }^{23}$ Details about the effect of the charge sign on the droplet size at which IEM dominates are presented in the study of Iribarne and Thomson. ${ }^{23}$

The polarity and structure of the liquid-vapor interface are essential elements of the surface reactivity and ion release mechanisms and as such have been heavily studied in planar interfaces. ${ }^{40-47}$ The empirical models of ion evaporation do not consider the complexity of the interface. In the ion-evaporation models ${ }^{23,24,39}$ the solvent effect is taken into account only via the Born solvation model. ${ }^{48} \mathrm{~A}$ more detailed description of the interface is considered in the equilibrium partition model (EPM) of C. Enke, which hypothesizes the existence of two regions in a droplet: a core region and an outer region that carries the surface charge. ${ }^{49,50}$ The model postulates that the species seen in the mass spectrum are those that make up the surface charge. Since EPM is a macroscopic qualitative model, it cannot directly provide quantitative measures such as the thickness and composition of the surface excess charge layer.

Gross et al. have proposed an intuitive qualitative model of how IEM may explain the charge states of macromolecules. ${ }^{26,51}$ This model suggests that the emission of small charge carriers from the surface of a droplet can occur when the electric field at the surface exceeds the critical electric field strength of a charge carrier, which depends on its solvation energy. Gross et al. hypothesis is discussed in the present article. Regarding macroscopic modeling, the solution of the Nonlinear Poisson-Boltzmann (NPB) equation for the charge distribution in a rigid spherical geometry generated by our group ${ }^{35}$ and, recently, 
by the Zare group ${ }^{52}$ are in excellent agreement.

The maximum amount of charge a drop can hold just before spontaneous fragmentation is estimated by the Rayleigh limit. ${ }^{38,53-56}$ We define this limit by using the Rayleigh fissility parameter $(X)$ given as

$$
X=\frac{Q^{2}}{64 \pi^{2} \gamma \varepsilon_{0} R^{3}} \approx\left(\frac{Q}{Q_{\mathrm{r}}}\right)^{2}
$$

where $Q$ is the droplet charge, $\gamma$ the surface tension, $\varepsilon_{0}$ and $R$ are the permittivity of vacuum and the radius of the droplet, respectively. When $X=1$ the system is at the Rayleigh limit. The droplet radius at the Rayleigh limit will be denoted as $R_{\mathrm{r}}$ and the corresponding charge $Q_{\mathrm{r}}$. Therefore, $X$ can also be expressed by the second equality in Eq. 1 . At $X<1$ the droplet is said to be "below the Rayleigh limit", while at $X>1$ is said to be "above the Rayleigh limit". When $X<1$ the drop is in a metastable state, the lifetime of which is determined by the degree of deviation from the Rayleigh limit. An uneven breaking of a drop is energetically more favorable. ${ }^{55}$

In the present analysis, we consider droplets that contain several single ions of different species with charge $\mathrm{e}^{+}$(where e denotes the elementary charge). In previous research we have identified the surface excess charge layer (SECL) and the maximum ion-concentration region (MICR). The trend that we have found in previous studies ${ }^{29,30}$ of droplets of different sizes and ions indicates that the presence of SECL with thickness $1.5 \mathrm{~nm}-2.0 \mathrm{~nm}$ is a robust feature that spans the entire range of droplet sizes including the micro-drops. Simulations of droplet sizes with a diameter $<16 \mathrm{~nm}$ with $\mathrm{Na}^{+}$ or $\mathrm{Cl}^{-}$ions have shown that $55 \%-24 \%$ (from the smaller to the larger droplet) of the ions reside in SECL. The charge distribution in SECL is the sum of the ion charge (free charge) and the solvent polarization. The larger the droplet, the smaller the effect of the ions in the solvent polarization.

\section{Models and Simulation Methods}

\section{Equilibrium simulations of droplets}

We performed molecular dynamics (MD) simulations of charged droplets comprised (a) water and $\mathrm{Na}^{+}, \mathrm{Cs}^{+}, \mathrm{Li}^{+}$and protonated histidine $\left(\mathrm{His}^{+}\right)$ions and (b) solvents with a dielectric constant less than that of water and $\mathrm{Na}^{+}$ions. Details of the systems are presented in Table 1. The simulations were performed by using the software NAMD version 2.12. ${ }^{57}$ Newton's equation of motion for each atomic site was integrated using the velocity-Verlet algorithm with a time step of $1.0 \mathrm{fs}$. The trajectories were analyzed using VMD 1.9.2. ${ }^{58}$ The duration of the production run is $40 \mathrm{~ns}$ for every system.

The water molecules were modeled with the TIP3P (transferable intermolecular potential with 3 points) ${ }^{59}$-CHARMM and the ions with the CHARMM36m ${ }^{60,61}$ force fields. The TIP3P-CHARMM is a m(odified)TIP3P, which is the original TIP3P with Lennard-Jones potential on the hydrogen sites. Hereafter, we will use the notation TIP3P for this water model. In the water-histidine systems the charge carriers are single protonated L-histidine mono-peptides, where the N-terminus is acetylated and the C-terminus is capped by $\mathrm{N}$ methylamide. In this work, we define the position 2 carbon on the imidazole ring as the location of the positive charge as it is approximately the center of the resonance structure.

The structure of sodiated drops comprised acetonitrile $(\mathrm{ACN})$, methanol $(\mathrm{MeOH}), 1,1$ dichloroethane (DCLE) and a mixture of wateracetonitrile was studied (Table 1). The number of molecules in these drops was determined so that for all droplets the equimolecular ra$\operatorname{dius}^{30}$ is $\approx 3.5 \mathrm{~nm}$. The organic molecules were modeled by using the Charmm GENeral Force Field. ${ }^{62}$ The dielecric constant and surface tension for the bulk solution were computed and are presented in Table S1 in SI.

In all the systems, all the forces were computed directly without any cut-offs. Equilibrium simulations in NAMD were set by placing the droplet in a spherical cavity of ra- 
dius $20.0 \mathrm{~nm}$ by using spherical boundary conditions. The cavity was sufficiently large to accommodate the shape fluctuation of the droplet. The droplet will eventually reach vapor pressure equilibrium. The systems were thermalized with Langevin thermostat with the damping coefficient set to $1 / \mathrm{ps}$. The Rayleigh limit of the droplet ( $X=1$ in Eq. 1) was calculated with the surface tension values of the water model used at the simulation temperature. ${ }^{63}$ Specifically, for TIP3P at $T=300 \mathrm{~K}$ the value of surface tension is taken to be $0.0523 \mathrm{~N} / \mathrm{m}$ and at $T=350 \mathrm{~K}$ to be $0.0432 \mathrm{~N} / \mathrm{m}$.

\section{Ion-ejection simulations from aqueous droplets with a mixture of ions}

Equilibrated droplets comprised $5880 \quad \mathrm{H}_{2} \mathrm{O}$ molecules - $10 \mathrm{Na}^{+}$ions - $10 \mathrm{Li}^{+}$(or $10 \mathrm{Cs}^{+}$) ions at temperatures $290 \mathrm{~K}, 300 \mathrm{~K}, 310 \mathrm{~K}$, $320 \mathrm{~K}, 330 \mathrm{~K}, 430 \mathrm{~K}$ were prepared. The ejection of the ions was examined by performing direct MD runs. 2-5 MD runs were performed at each temperature. At $T=290 \mathrm{~K}$ no ion ejection was observed over several nanoseconds of simulations. The software used and details of the MD runs are the same as that described in Sec. "Equilibrium simulations of droplets" except that the electrostatic interactions were treated with the multilevel summation method. ${ }^{64}$

\section{Droplet disintegration simulations at elevated temperature}

Evaporation runs were performed for four systems, for which their initial configurations comprised (a) $\sim 3 \times 10^{4} \mathrm{H}_{2} \mathrm{O}$ molecules, $67 \mathrm{Na}^{+}$ions and $23 \mathrm{Cl}^{-}$ions; (b) same as (a) but with $\mathrm{Li}^{+}$ ions; (c) $\sim 4 \times 10^{3} \mathrm{H}_{2} \mathrm{O}$ molecules - $13 \mathrm{Na}^{+}$ ions; (d) same as (c) but with $\mathrm{Li}^{+}$ions. The initial configurations were equilibrated at $350 \mathrm{~K}$ within a spherical cavity of radius $20 \mathrm{~nm}$. The system was re-thermalized at $450 \mathrm{~K}$ before the spherical cavity was removed to let the droplet evaporate in vacuo. The system temperature was maintained at $450 \mathrm{~K}$ for the duration of the production run. The software used and details of the MD runs are the same as that described in Sec. "Equilibrium simulations of droplets".

\section{Results and Discussion}

\section{Abundance of ions in the surface excess charge layer}

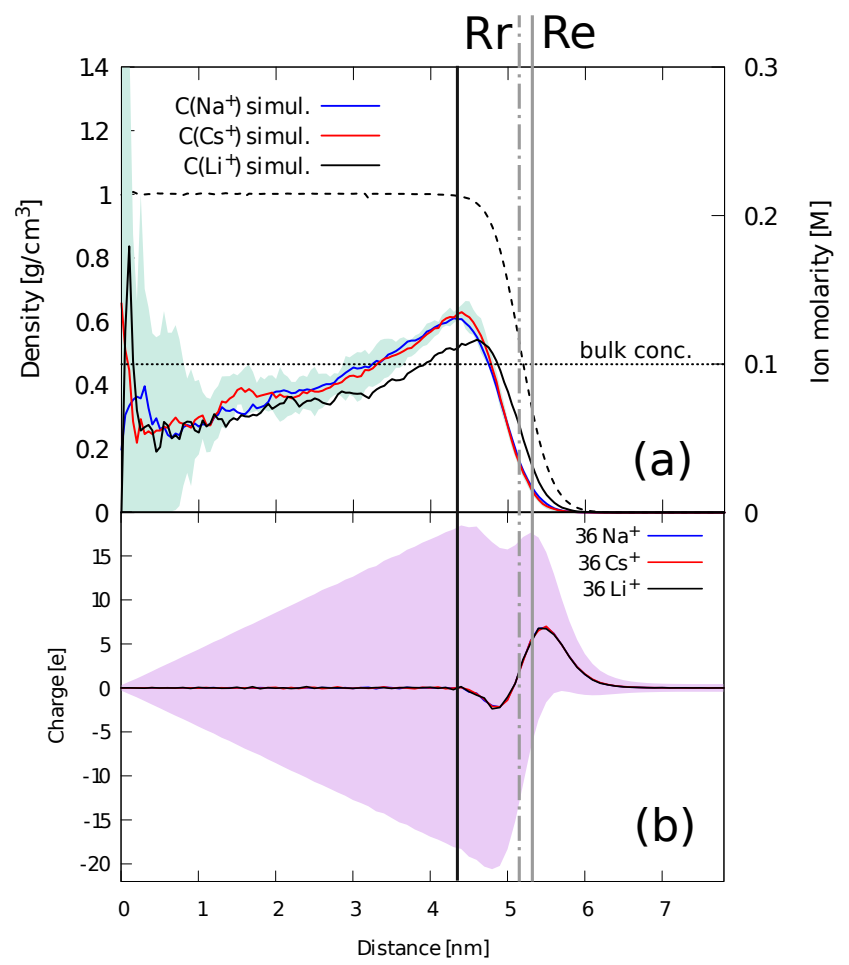

Figure 2: (a) $\mathrm{Na}^{+}$(blue), $\mathrm{Cs}^{+}$(red), $\mathrm{Li}^{+}$(black) radial concentration profiles vs distance from the droplet COM for systems comprised $2 \times 10^{4}$ $\mathrm{H}_{2} \mathrm{O}$ molecules and 36 ions at $T=350 \mathrm{~K}$. The water density is shown by the dashed line and measured in the left axis. The vertical black line marks the distance at which the charge distribution starts to build-up. The horizontal line shows the bulk concentration. $R_{\mathrm{e}}$ and $R_{\mathrm{r}}$ denote the equimolecular and Rayleigh radius, respectively. (b) Total charge (from the hydrogen, oxygen and ion sites) distribution as a function of distance from the drop COM.

Figure 2 (a) shows the radial distribution profiles of ions and water molecules in droplets comprised $2 \times 10^{4} \mathrm{H}_{2} \mathrm{O}$ molecules and $36 \mathrm{Na}^{+}$, 
Table 1: Systems studied, characteristic dimensions and concentrations. $n_{\mathrm{H}_{2} \mathrm{O}}$ denotes average number of water molecules and $n_{\mathrm{I}}$ number of ions in the droplet during the production runs. $R_{\mathrm{e}}[\mathrm{nm}]$ is the equimolecular radius, computed by using density of the TIP3P model at $350 \mathrm{~K}$ to be $0.9539 \mathrm{~g} / \mathrm{cm}^{3} \cdot r_{\max }[\mathrm{nm}]$ is the distance from the droplet COM to the maximum of the ion concentration profile. $\lambda_{\mathrm{PB}}$ is given in Eq. 2, and the dielectric constant was computed for the solvents used (Table S1). $X$ is the fissility parameter of the simulated droplet (Eq. 1). "Range" [nm] denotes the interval defined from the droplet COM in which the surface excess charge is located. The outer boundary of the interval is at water density of $5 \times 10^{-4} \mathrm{~g} / \mathrm{cm}^{3}$. Since the outer boundary corresponds to a specific density, there is no error bar associated with it. $n_{\text {out }}$ is the number of ions in the surface excess charge layer. Details are presented in the text. $C_{\mathrm{SECL}}$ [Molarity] is the ion concentration in the surface excess charge layer.

\begin{tabular}{ccccccccc}
\hline$n_{\mathrm{H}_{2} \mathrm{O}}$ & $n_{\mathrm{I}}$ & $R_{e}$ & $X$ & $r_{\max }$ & $\lambda_{\mathrm{PB}}$ & Range & $n_{\text {out }}$ & $C_{\mathrm{SECL}}(\mathrm{mol} / \mathrm{L})$ \\
$6 \times 10^{3}$ & $19 \mathrm{Na}^{+}$ & 3.55 & 0.85 & $2.65-2.70$ & 1.41 & $2.6 \pm 0.1-4.4$ & $10.6 \pm 0.6$ & $0.062 \pm 0.004$ \\
$6 \times 10^{3}$ & $16 \mathrm{His}^{+}$ & 3.55 & 0.62 & $2.85-2.90$ & 1.68 & $2.8 \pm 0.1-4.6$ & $8.4 \pm 0.6$ & $0.044 \pm 0.002$ \\
\hline $2 \times 10^{4}$ & $36 \mathrm{Na}^{+}$ & 5.31 & 0.92 & $4.30-4.35$ & 1.67 & $4.4 \pm 0.1-6.1$ & $13.5 \pm 1.0$ & $0.038 \pm 0.003$ \\
$2 \times 10^{4}$ & $36 \mathrm{Cs}^{+}$ & 5.31 & 0.92 & $4.40-4.45$ & 1.67 & $4.4 \pm 0.1-6.1$ & $13.7 \pm 1.0$ & $0.038 \pm 0.003$ \\
$2 \times 10^{4}$ & $35 \mathrm{Li}^{+}$ & 5.31 & 0.90 & $4.60-4.65$ & 1.72 & $4.4 \pm 0.1-6.1$ & $15.8 \pm 0.8$ & $0.044 \pm 0.002$ \\
\hline \hline $2000 \mathrm{ACN}$ & $14 \mathrm{Na}^{+}$ & 3.47 & 0.66 & $2.50-2.55$ & 0.323 & $2.4 \pm 0.1-4.6$ & $9.6 \pm 0.6$ & $0.045 \pm 0.003$ \\
$1500 \mathrm{ACN}-$ & $16 \mathrm{Na}^{+}$ & 3.47 & 0.86 & $0.80-0.85$ & 0.512 & - & - & - \\
$1500 \mathrm{TIP} 3 \mathrm{P}$ & & & & & & & & - \\
$1300 \mathrm{DCLE}$ & $9 \mathrm{Na}^{+}$ & 3.49 & 0.40 & $2.50-2.55$ & 0.192 & - & - & - \\
$2600 \mathrm{MeOH}$ & $13 \mathrm{Na}^{+}$ & 3.47 & 0.72 & $2.60-2.65$ & 0.466 & $2.4 \pm 0.1-4.6$ & $9.3 \pm 0.6$ & $0.044 \pm 0.003$ \\
\hline \hline
\end{tabular}

$36 \mathrm{Cs}^{+}$and $36 \mathrm{Li}^{+}$ions. The profiles have been normalized by dividing the raw histogram data with the volume of a spherical shell $\left(\frac{4}{3} \pi[(r+\right.$ $d r)^{3}-r^{3}$ ] where $r$ is the distance from the droplet COM). Toward the interior, the ion distribution is expected to show an exponential decay as it is predicted by the solution of the NPB for a rigid spherical droplet. ${ }^{28,29,35}$ The characteristic decay length ${ }^{28,29,35}$ is expressed as

$$
\lambda_{\mathrm{PB}} \approx \frac{\epsilon k_{\mathrm{B}} T}{\sigma}
$$

where $k_{B}$ is Boltzmann constant, $T$ is temperature, $\epsilon$ is the permittivity of water and $\sigma=\frac{|Z| e^{2}}{4 \pi R_{\mathrm{e}}^{2}}$ for ions of charge $\pm 1 e$, where $e$ is the charge of the electron, $|Z|$ is the absolute value of droplet charge in units of $e$. Here the assumption is that there are no counterions. For the droplet comprised $3 \times 10^{4} \mathrm{H}_{2} \mathrm{O}$ molecules and $44 \mathrm{Na}^{+}$ ions (radius equal to $6.08 \mathrm{~nm}$ ) $\lambda_{\mathrm{PB}}=1.08 \mathrm{~nm}$ if we consider a dielectric constant (experimental) $\varepsilon \approx 61.7$ at $T=350 \mathrm{~K}$.

A common feature in all the distributions is that they initially show an exponential decay from their maximum (at $r_{\max }$ ) that can be fitted reasonably well by (const) $\exp \left(-r / \lambda_{\mathrm{PB}}\right)$ where $r$ is the distance from the droplet COM. Towards, the interior the decay is much slower. The maximum of the $\mathrm{Li}^{+}$distribution is more distant from the droplet COM than those of $\mathrm{Na}^{+}$and $\mathrm{Cs}^{+}$ions. We attribute this difference to the fact that $\mathrm{Li}^{+}$tightly binds its first hydration shell, thus the ion is effectively larger than the $\mathrm{Na}^{+}$or $\mathrm{Cs}^{+}$ions. ${ }^{65-70}$ The horizontal line (Fig. 2 (a)) marks the bulk concentration, which is simply estimated by the number of ions divided by the volume of the droplet (with $R_{\mathrm{e}}$ radius).

The decay of the ion distribution for $r>r_{\max }$ is mainly due to shape fluctuations. In previous research $^{28}$ we have presented the fitting of this region by a function that takes into account the droplet shape fluctuations, the ion radius and $\lambda_{\mathrm{PB}}$. Because of the role of the shape fluctuations we have suggested that the ion distribution may be viewed better from the droplet 


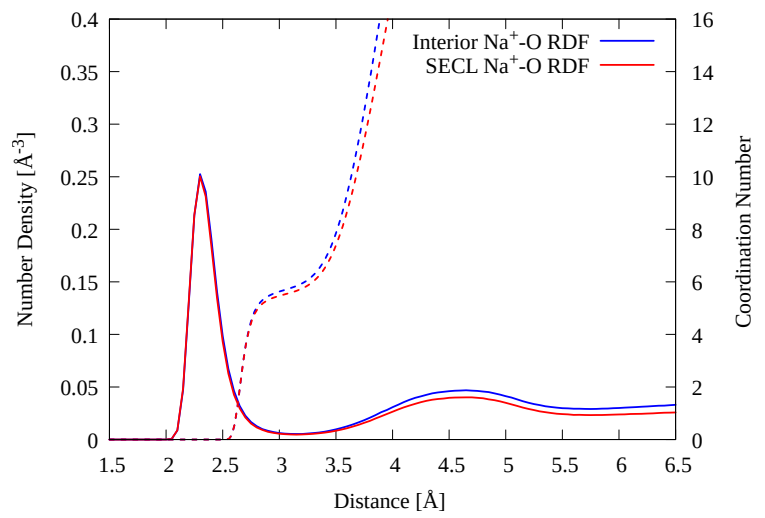

(a)

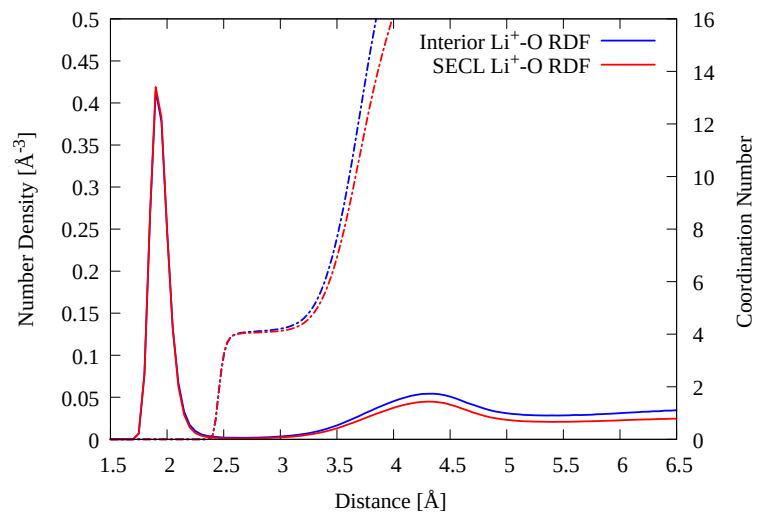

(b)

Figure 3: (a) Radial distribution function (RDF) (measured in the left $\mathrm{y}$-axis as one views the figure) and its integral (measured in the right y-axis) of $\mathrm{Na}^{+}$-Oxygen site of $\mathrm{H}_{2} \mathrm{O}$ and (b) of $\mathrm{Li}^{+}$-Oxygen site of $\mathrm{H}_{2} \mathrm{O}$ in the surface excess charge layer and droplet interior. The droplet comprises $5880 \mathrm{H}_{2} \mathrm{O}$ molecules and the ions. The bin size of the RDF is $0.05 \AA$ and the plots are normalized by dividing the raw data by the number of ions in each bin and the bin size.

surface, because in that way the depth of the ion from the droplet surface can be detected. ${ }^{28}$

Figure 2 (b) shows the radial total charge distribution. The vertical black line marks the distance at which the charge distribution starts to build-up. We find that for $\mathrm{Na}^{+}$and $\mathrm{Cs}^{+}$ions the $62 \%$ of the total number of ions reside in the maximum ion-concentration region, which is in the interval $3.3 \mathrm{~nm}-4.7 \mathrm{~nm}$. The excess number of ions relative to the bulk value is 3.5 ions. For $\mathrm{Li}^{+}$ions, the maximum ion-concentration region (which is in the interval $4.0 \mathrm{~nm}-4.9 \mathrm{~nm}$ ) in- cludes $41 \%$ of the total number of ions. Lithium concentration is lower than that of $\mathrm{Na}^{+}$and $\mathrm{Cs}^{+}$ ions because it has a higher concentration in the surface excess charge layer.

The surface excess charge layer starts approximately at the maximum of the ion distribution for $\mathrm{Na}^{+}$and $\mathrm{Cs}^{+}$and includes $38 \%$ of the ions. The surface excess charge layer for $\mathrm{Li}^{+}$ ions includes the maximum of the ion distribution and $45 \%$ of the ions. The combination of data from previous studies ${ }^{29}$ and the current study shows that the larger the droplet, the smaller the concentration of ions in maximum ion-concentration region and in the surface excess charge layer when counterions are not present.

Often, the width of the air-water interface is described by the "10-90" thickness rule. ${ }^{71}$ For pure water it is approximately $3 \AA-4 \AA .{ }^{72}$ In the charged droplets the width of this region is $\approx 8 \AA$ and it is determined by shape fluctuations. The width of the surface excess charge layer is broader than the 10-90 interface. In order to examine the effect of the suppression of the shape fluctuations we performed simulations under confinement of a liquid with ions in a spherical rigid geometry (Fig. S1 in SI). The ion decay toward the droplet interior appears to be somewhat affected by the suppression of the fluctuations. The effect of the fluctuations in the decay of the ion distribution towards the droplet interior will be more pronounced in smaller droplets.

The radial ion distribution profile of a droplet that is composed of $6 \times 10^{3} \mathrm{H}_{2} \mathrm{O}$ molecules - 16 $\mathrm{His}^{+}$ions was also computed (Fig. S2 in SI). Simulations of the droplets were initiated with $19 \mathrm{His}^{+}$ions. Differently from the corresponding sodiated droplets, several $\mathrm{His}^{+}$ions were quickly ejected from the droplet leading to a long-living metastable state with $16 \mathrm{His}^{+}$ions. The fewer $\mathrm{His}^{+}$ions than $\mathrm{Na}^{+}$ions in a droplet of the same size indicates that $\mathrm{His}^{+}$reduces the surface tension of the aqueous droplets more than the $\mathrm{Na}^{+}$ions. The profile of $\mathrm{His}^{+}$shows that the ions are accumulated in the maximum ion-concentration region.

In order to examine whether there is a difference in the solvation of ions in the surface 
excess charge layer and droplet interior, the radial distribution function (RDF) between $\mathrm{Li}^{+}$oxygen site of $\mathrm{H}_{2} \mathrm{O}$ and $\mathrm{Na}^{+}$-oxygen site of $\mathrm{H}_{2} \mathrm{O}$ were computed (Fig. 3). Figure 3 shows that there is no difference in the first hydration shell of the ions in the two locations, but there are some small differences in the second shell. The second solvation shell has contributions from the $\mathrm{H}_{2} \mathrm{O}$ molecules found in the droplet interior and those found in the outer droplet layers. The similarities in the RDFs strengthen the argument that the decay that we see in the solvent density and ion density in the surface excess charge region is mainly due to shape fluctuations. These differences have to be examined more carefully by including in the force fields subtle details due to electronic polarization. This is a question that we continue to explore along the lines of the presented analysis.

\section{Ejection mechanism}

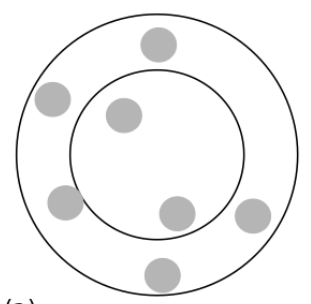

(a)
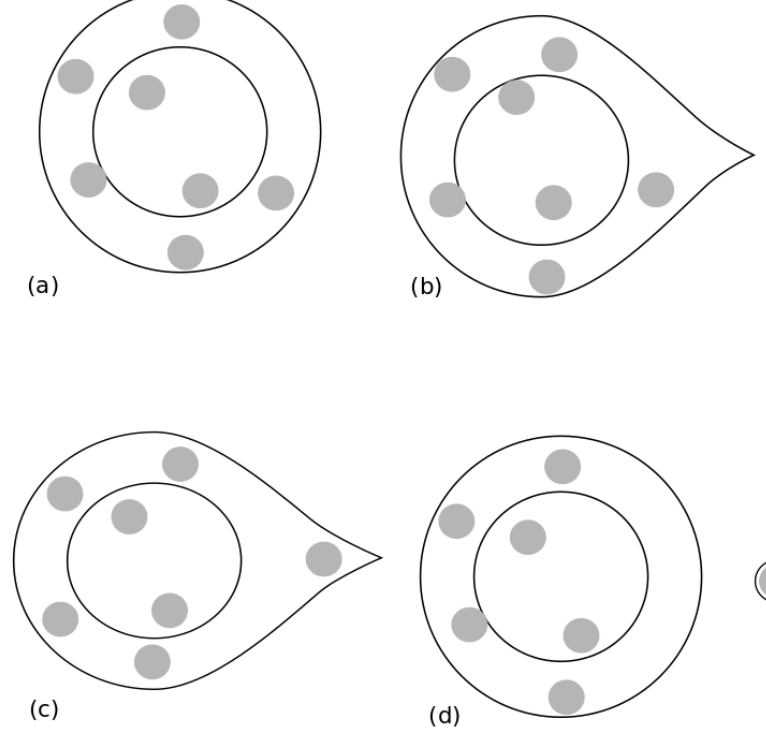

Figure 4: (a)-(d) Schematic sequence of events that lead to the ion release. The grey spheres represent the ions. The region between the internal circle and the outer line represents the surface excess charge layer (details in the text).

The different locations of ions in the surface excess charge layer raises the question of how

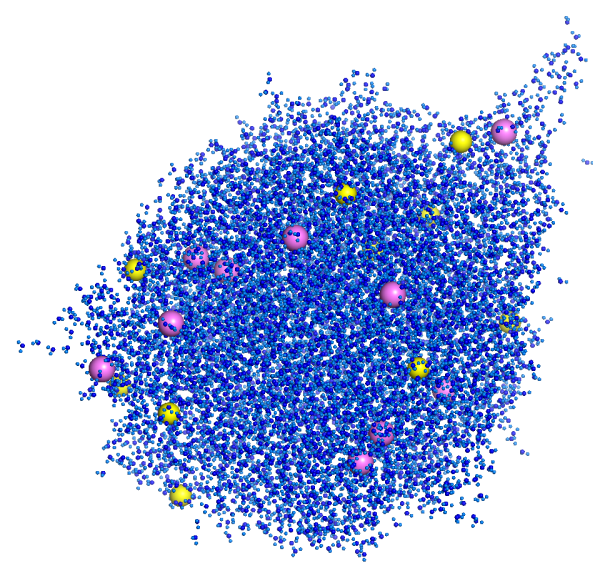

Figure 5: A typical snapshot of a droplet with an emerging conical fluctuation. The droplet comprises 5880 TIP3P water molecules, $10 \mathrm{Cs}^{+}$ ions (shown in violet) and $10 \mathrm{Na}^{+}$ions (shown in yellow).

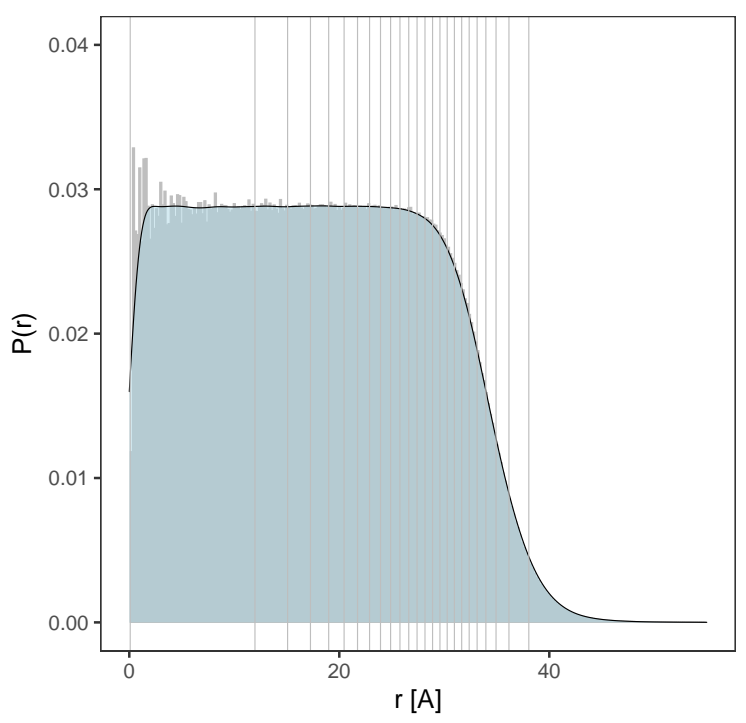

Figure 6: The normalized radial distribution function of the centres of mass of the water molecules corresponding the the system shown in Fig. 5. 


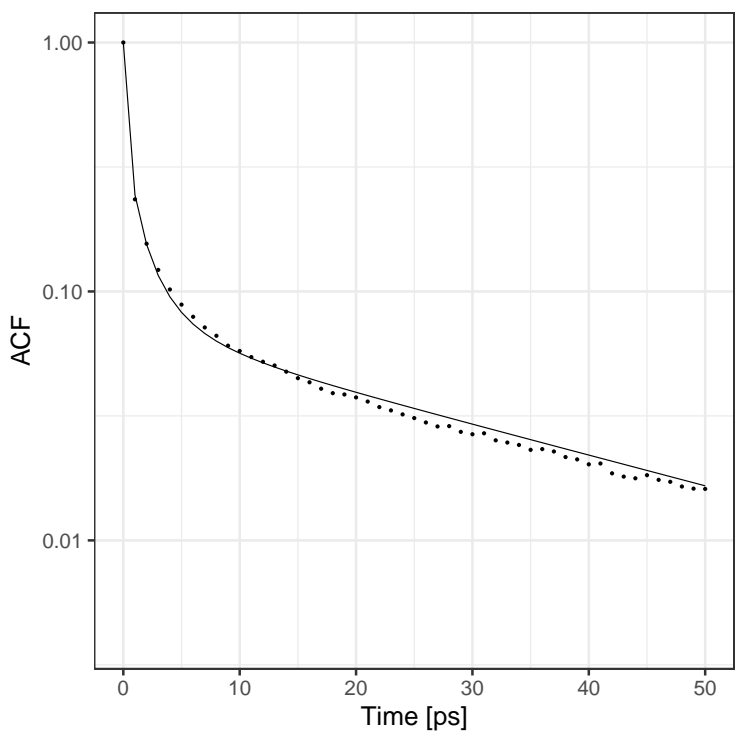

Figure 7: Autocorrelation function $(\mathrm{ACF})$ of a shell state variable. The decay is the result of molecule diffusion across the shell boundaries as well as transfer across shells due to some other mechanisms.

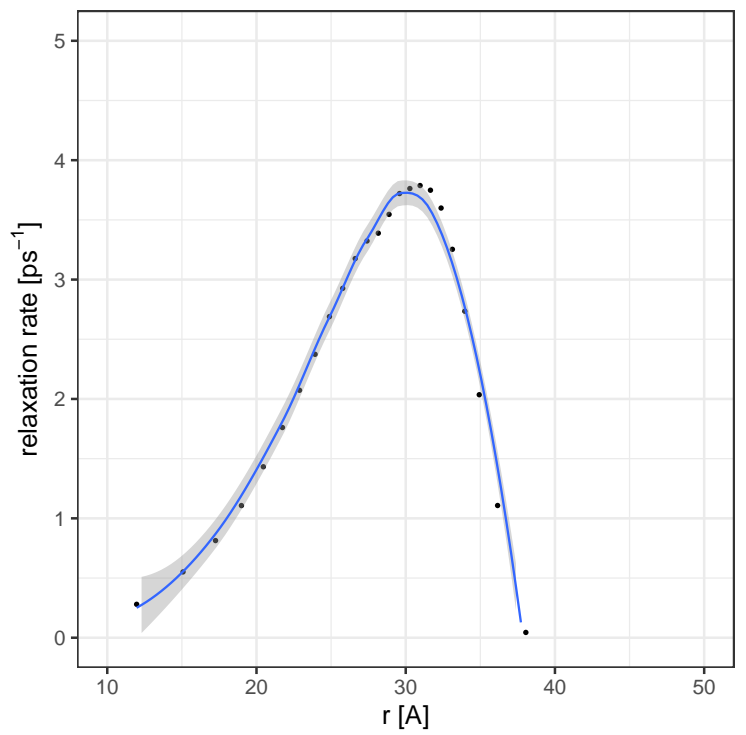

Figure 8: The first relaxation rate of the $\mathrm{ACF}$ shown in Fig. 7 as a function of shell position (details are presented in the text). the equilibrium structure is related to their order of ejection. To examine this question we performed simulations of aqueous droplets with mixtures of ions at $T=300 \mathrm{~K}, 310 \mathrm{~K}, 320 \mathrm{~K}$ and $330 \mathrm{~K}$. The simulations consistently showed that solvated $\mathrm{Li}^{+}$ions are ejected first. The ejections take place as a single solvated $\mathrm{Li}^{+}$ion at $T=300 \mathrm{~K}$ and $310 \mathrm{~K}$. At $T=320 \mathrm{~K}$ it is possible that two solvated $\mathrm{Li}^{+}$ions are released almost simultaneously from diametrically opposed conical protrusions. A typical snapshot of concurrent ejections of two solvated $\mathrm{Li}^{+}$ions is shown in Fig. S3 in SI. The formation of the two opposed cones is reminiscent of the jets that have been observed in Rayleigh fission. ${ }^{73-75}$ The droplets that we study are not large enough to demonstrate the continuous ejection of ions from the cones as it is found in experiments. ${ }^{73-75}$ We attribute the formation of two cones to the fact that at $T=330 \mathrm{~K}$, the surface tension of the systems is reduced (relative to that at $T=310 \mathrm{~K}$ ), which may render the system slightly above the Rayleigh limit. At $T=430 \mathrm{~K}$, out of 5 runs, 3 of them had $\mathrm{Li}^{+}$ion ejected first, and 2 of them had $\mathrm{Na}^{+}$ion ejected first. We infer that, the higher the temperature the less the nature of the ion affects the order of ejection.

Simulations of droplets with a mixture of $\mathrm{Na}^{+}$ and $\mathrm{Cs}^{+}$ions show that they are released with almost the same frequency $\left(\mathrm{Na}^{+}\right.$is released first marginally more frequently than $\mathrm{Cs}^{+}$), which may be a consequence of the identical ion distribution profiles (Fig. 2).

In summary, the simulations indicate that at the lower temperature the ions in abundance within the surface excess charge layer are released first. Contrary to the frequently used non-equilibrium molecular dynamics trajectories in computations related to the analysis of the mass spectra signals, the present study demonstrates the significance of the droplet equilibrium structure in determining the ion ejection mechanisms. Our findings are consistent with the EPM principles. EPM does not directly consider the temperature effect, which may change the statistics of which ion is ejected first. The temperature effect is considered in the atomistic simulations. 
Now, we analyze the ejection mechanism. A charged droplet close to the Rayleigh limit spontaneously develops protuberances similar to the Taylor cones. ${ }^{76}$ These solvent shape fluctuations are persistent structures that may or may not contain charged species. The cones are in general the locations from where the ions are ejected.

The ejection mechanism follows distinct steps, shown schematically in Fig. 4. The droplet undergoes large shape fluctuations that involve the formation of transient cones (Fig. 4 (b)). The cones are the results of global shape fluctuations. The global nature of the shape fluctuation is evidenced by the fact that if the electrostatic forces are truncated to a distance smaller than the droplet radius, the conical shapes do not appear. An ion may diffuse within the cone (Fig. 4 (c)). Once the ion diffuses within the cone it travels toward the tip from where it is released as a small cluster of the solvated ion (Fig. 4 (d)). On the average, $\mathrm{Li}^{+}$is released with $17 \mathrm{H}_{2} \mathrm{O}$ molecules, $\mathrm{Na}^{+}$ with 14.8 and $\mathrm{Cs}^{+}$with 16 .

In summary, ion ejection takes place when there is a co-operation of events: a cone appears in a location where within its life-time an ion can enter it either by diffusion or by shape fluctuations that engulf the ion in the cone.

We have analyzed the dynamics of the protuberances shown in Fig. 5. The droplet comprises 5880 TIP3P $\mathrm{H}_{2} \mathrm{O}$ molecules, $10 \mathrm{Cs}^{+}$ions (shown in violet) and $10 \mathrm{Na}^{+}$ions (shown in yellow). The system temperature is kept at $330 \mathrm{~K}$. A typical conical formation is clearly seen in the upper right corner of the snapshot. Two ions are present at the foundation of the cone. Monitoring of this cone shows its disappearance within 20 ps without subsequent ion ejection event.

In Fig. 6 we plot the radial distribution function (RDF) of the water molecules' centre of mass obtained during a segment of 1 ns production run. No ion evaporation events were observed during this run. The RDF shows the uniform solvent distribution in the core of the droplet and tapered slope at the edge of the droplet. The slope is the result of the shape fluctuations of the droplet and does not indicate a decrease of the solvent density close to the surface. The vertical lines indicate separation of the droplet in equimolar shells at $\left\{R_{1}, \ldots, R_{n}\right\}$. Every shell $R_{k} \leq\|\mathbf{r}\| \leq R_{k+1}$ contains the same number of molecules.

We constructed a state variable for each of the shells

$$
A_{k}\left(\mathbf{x}_{i}(t)\right)= \begin{cases}1 & R_{k} \leqslant\left\|\mathbf{r}(t)_{i}\right\| \leqslant R_{k+1} \\ 0 & \text { otherwise }\end{cases}
$$

where $\mathbf{x}_{i}$ is the position of the center of mass of an i-th solvent molecule. We calculated the corresponding auto-correlation functions

$$
\mathrm{ACF}_{k}(\tau)=\left\langle A_{k}\left(\mathbf{x}_{i}(t+\tau)\right) A_{k}\left(\mathbf{x}_{i}(t)\right)\right\rangle
$$

A typical auto-correlation function corresponding the region close the droplet surface is plotted in Fig. 7. We numerically fitted the decay function to the following approximation using the $\mathrm{R}$ statistical analysis package ${ }^{77}$

$$
\mathrm{ACF}_{k}(\tau) \sim c e^{-\sqrt{r_{1} \tau}}+(1-c) e^{-r_{2} \tau}
$$

The first term corresponds to a diffusive transfer of molecules across the shell boundaries and the second term corresponds to a regular Markov process. The fit captures the general shape of the ACF decay indicating that the above two mechanisms account for the solvent transfer between the shells.

In Fig. 8 we plot the first relaxation rate $r_{1}$ as a function of the shell position. In the plot, we observe that for the region, where the conical shape fluctuations are observed, the first relaxation rate has small values. This may account for a long life of the emerging protuberances.

The finding of the cones is consistent with our previous research where we approached the problem of the ion release from droplets using a different methodology. ${ }^{27}$ In that study, we computed the free energy of detachment of a solvated ion from a parent drop along a collective reaction coordinate, which takes into account the position of all the solvent molecules and ions. We found that an ion may be ejected from a conical formation that corresponds to the barrier top of the free energy profile along this reaction coordinate. ${ }^{27,78}$ 
Generally, from the simulation studies we cannot infer whether the release of ions from minute nanodroplets follows the ion evaporation mechanism ${ }^{23-25}$ (IEM) or a Rayleigh fission. It has been found in experiments that Rayleigh fission of aqueous microdrops releases $20 \%-40 \%$ of charge. ${ }^{79}$ In a droplet of up to a few thousands of water molecule, this percentage corresponds to only a few ions. The scaling of the data implies that the observation of the single-ion ejection from a minute nanodrop does not warrant an IEM mechanism. Our view is different from that that has been presented in the literature ${ }^{80,81}$ where the ejection of simple ions from droplets composed of $\approx 1000 \mathrm{H}_{2} \mathrm{O}$ is considered evidence of the ion evaporation mechanism (in the sense of Iribarne-Thompson and Labowsky et al.). We elaborate more on this point in the next section. We have also observed in simulations (see Table 1 and Ref. ${ }^{29}$ ) and we justify it in the Appendix that the smaller a droplet the smaller the $X$ value in which it settles in a quasiequilibrium (metastable) state. The fact that a droplet of a few thousand of water molecules ejects ions below the Rayleigh limit, is consistent with the Rayleigh fluctuations (see Appendix), therefore, the ejection of ions cannot be differentiated from a Rayleigh mechanism. The fact that the smaller the droplet, the smaller the $X$ value of a long-living metastable state may explain the charge state of small globular proteins. It has been reported in the mass spectrometry literature, that the charge state of small proteins such as ubiquitin, cytochrome $\mathrm{C}$, ovalbumin is less than the Rayleigh charge of a droplet of the same size. ${ }^{82}$ The size of these proteins surrounded by approximately a layer of $\mathrm{H}_{2} \mathrm{O}$ molecules is similar or smaller to that of a droplet that is composed of $\approx 6 \times 10^{3} \mathrm{H}_{2} \mathrm{O}$ molecules. As shown in Table 1, a droplet of this size is found in a long-living metastable state at $X \approx 0.85-0.6$ (vs. $X \approx 0.90-0.95$ in the much larger droplets). The Rayleigh fluctuations justify a smaller charge state of a protein that can be acquired via a charged-residue mechanism.
Solvents with $\varepsilon<\varepsilon_{H_{2} O}$

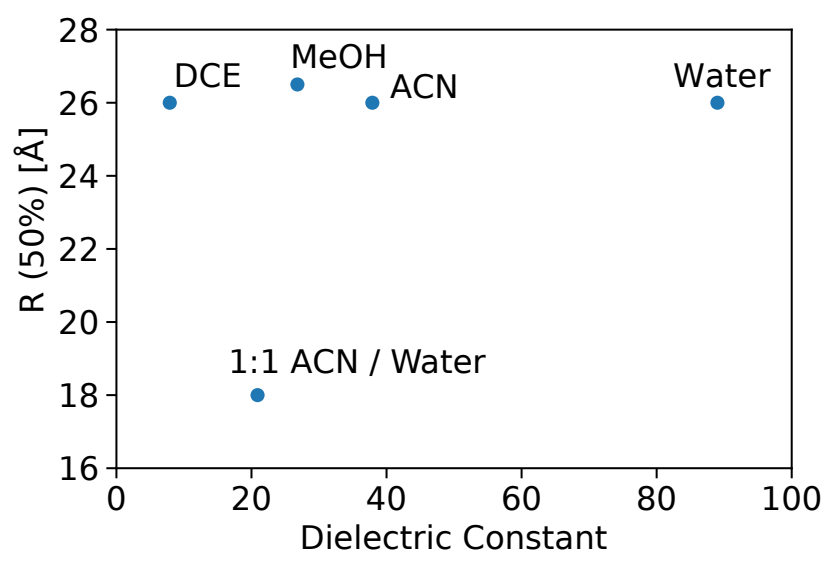

Figure 9: Distance from the droplet COM $(R(50 \%))$ that divides in half the number of ions as a function of the solvent's dielectric constant. The systems are presented in Table 1.

The $\mathrm{Na}^{+}$radial distributions were computed in droplets composed of solvent with lower dielectric constant than water. The systems are shown in Table 1. All the droplets have approximately the same $R_{e}$. Among all singlesolvent droplets we have tested, the solvent density reduces to $5 \times 10^{-4} \mathrm{~g} / \mathrm{cm}^{3}$ at approximately $1.9 \mathrm{~nm}$ from the $\mathrm{Na}^{+}$concentration maximum. As shown in Fig. 9, regardless of the dielectric constant of the solvent, the distance from the droplet's COM at which the $50 \%$ of the ions are found is the same.

The charge distribution profiles for acetonitrile, methanol and 1,1-dichloroethane droplets are presented in Fig. S4, Fig. S5, and in Fig. S6 in SI, respectively. In the acetonitrile and the methanol droplets, the charge distribution profile undulates when the solvent density sharply declines similar to that shown in the lower panel of Fig. 10 (a). Differently, the charge distribution profile of 1,1-dichloroethane droplet shows only a maximum at $3.2 \mathrm{~nm}$.

Figure 10 (a) - upper panel shows the radial ion distribution, the acetonitrile partial density profile, the water partial density profile and the $\mathrm{Na}^{+}$charge profile for a droplet of 1500 $\mathrm{CH}_{3} \mathrm{CN}$ molecules, $1500 \mathrm{H}_{2} \mathrm{O}$ molecules and $16 \mathrm{Na}^{+}$ions. The partial density of water is calculated by considering only water molecules and excluding the acetonitrile molecules in the 


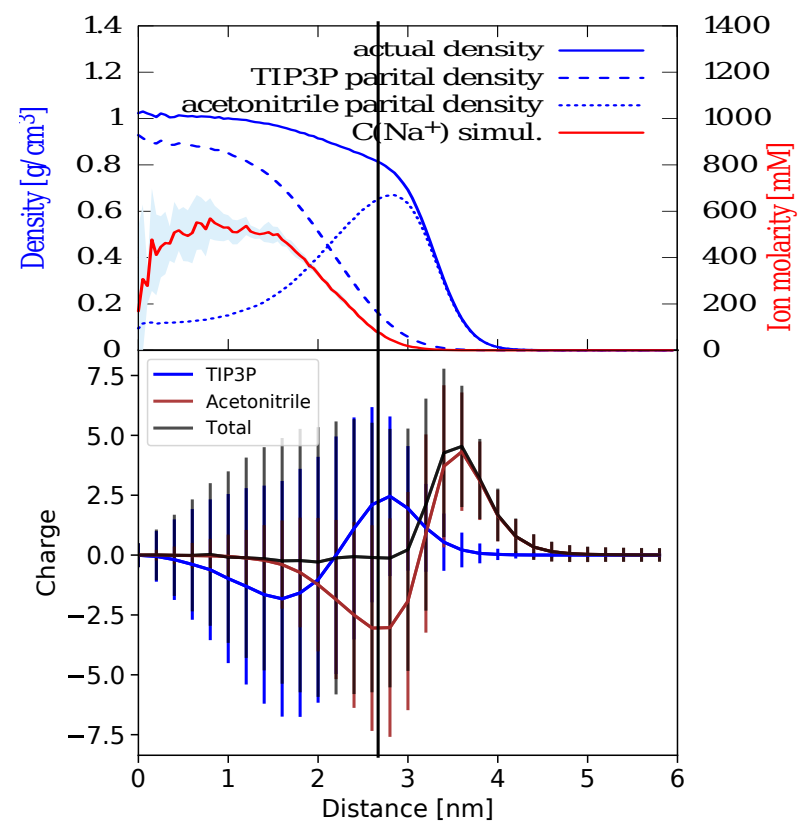

(a)

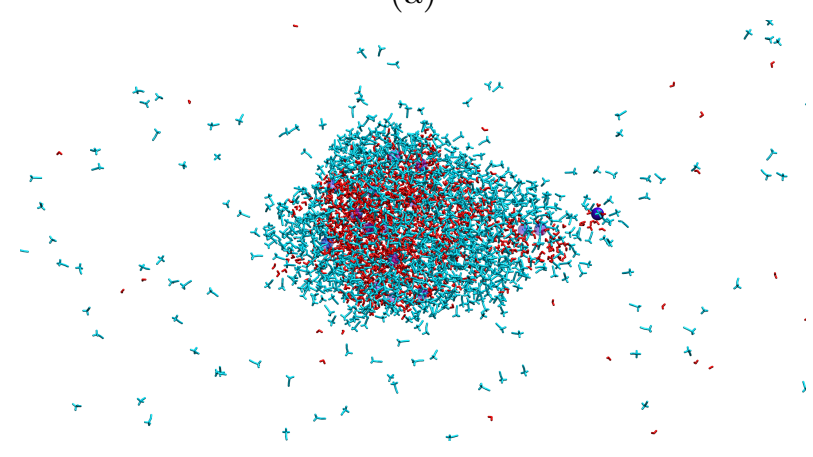

(b)

Figure 10: (a) Same as Fig. 2 but for a drop $\left(R_{e} \approx 3.5 \mathrm{~nm}\right)$ comprising acetonitrile, water molecules and sodium ions at 300 K. (b) Typical snapshot of $\mathrm{Na}^{+}$(blue sphere) ejection from a $\mathrm{H}_{2} \mathrm{O}$ (red colored core)-ACN (light blue colored outer layer) droplet.

computation of density, and vice versa. Therefore the actual density is the sum of the partial densities of TIP3P and acetonitrile. The partial density of $\mathrm{H}_{2} \mathrm{O}$ in the interior part of the droplet is higher than in the outer part, while the density of $\mathrm{CH}_{3} \mathrm{CN}$ follows the opposite trend. Contrary to the acetonitrile droplets, the $\mathrm{Na}^{+}$ions tends to be located in the interior part of the droplet and its concentration reaches its maximum at about $0.8 \mathrm{~nm}$ from the COM, where the partial density of water is higher than the partial density of acetonitrile. The density in this droplet dies off to $5 \times 10^{-4}$ $\mathrm{g} / \mathrm{cm}^{3}$ at approximately $3.7 \mathrm{~nm}$ from the $\mathrm{Na}^{+}$ concentration maximum.

Figure 10 (a)-lower panel shows the charge distribution profile in the droplet. The total profile is dominated by a positive peak. An incipient negative trough appears at $\approx 2.8 \mathrm{~nm}$.

The ejection of ions is shown in Fig. 10 (b). The water molecules make a path within the $\mathrm{ACN}$ for the ion to be released.

\section{Limitations of atomistic sim- ulations in detecting the IEM}

Simulations were performed at elevated temperature in order to examine the relation between the ion-ejection mechanism and the structure of the droplet outer layer when the solvent evaporation rate is higher than the ion diffusion rate. Accumulation of analytes on the droplet surface because of rapid solvent evaporation has often been speculated to explain the mechanisms by which the species detected in mass spectrometry are formed. ${ }^{83}$ Higashi et al. have performed atomistic simulations at $T=460 \mathrm{~K}$ of charged nanodrops comprised 2500 and 1000 $\mathrm{H}_{2} \mathrm{O}$ molecules, $\mathrm{Na}^{+}$ions and $\mathrm{Cl}^{-}$ions in order to provide direct evidence of the IEM. ${ }^{80}$ Temperature determines to a great extent the events in a droplet's lifetime. Even though temperature of $T=460 \mathrm{~K}$ in simulations appears to be high it is still not clear whether it is unrealistic because of conflicting experimental data on droplet temperature. ${ }^{84,85}$

In general, droplet temperature will depend on the details of the instrument and the specific experiment. A droplet within a background gas and a partial pressure less than the equilibrium vapour pressure, will cool down due to evaporation. Antoine et al. have found by using laser-induced fluorescence and Mie scattering measurements that the temperature of electrosprayed microdrops increases to $307 \mathrm{~K} .{ }^{84}$ The increase is attributed to the conductive thermal 
transfer with the sheath gas. Cook et al. use a different experimental set-up and they find that microdrops cool initially by $30 \mathrm{~K} .{ }^{85}$ Beauchamp et al. have noted that time required for the velocity redistribution between the droplet outer layers and deep interior may lead to a colder surface. ${ }^{79,86}$ In the small nano-drops, the velocity redistribution will be rapid, thus a uniform temperature can be established.

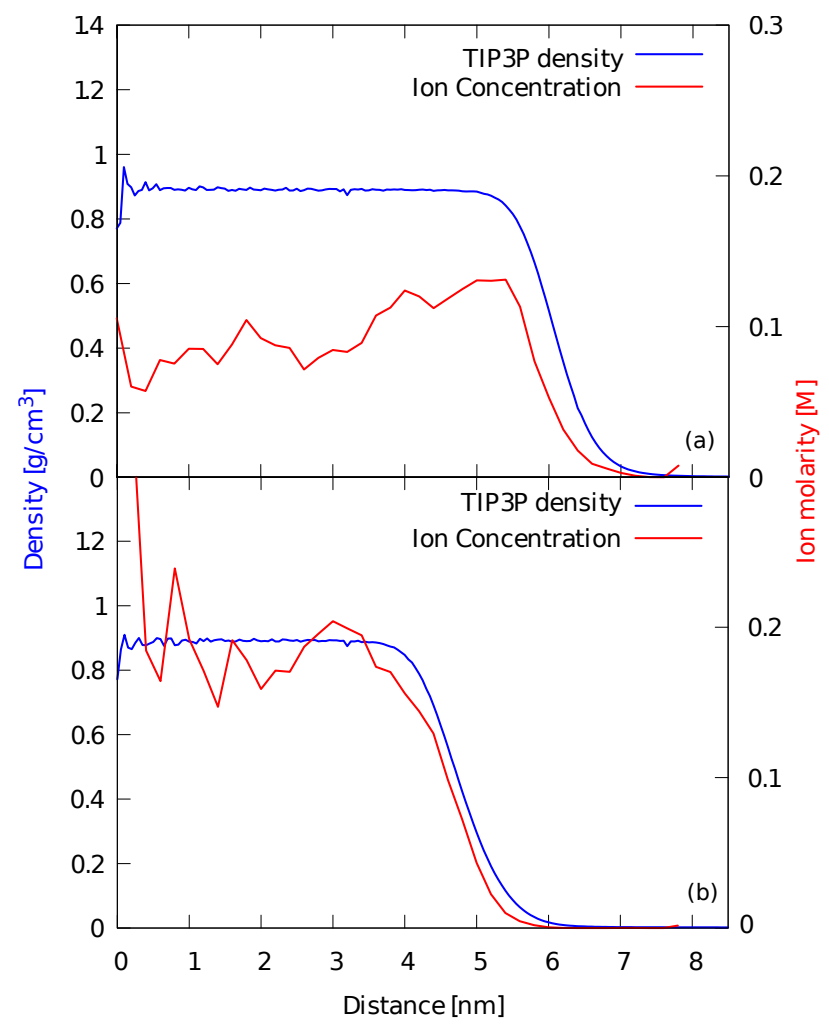

Figure 11: Water density and ion concentration profile of the droplet comprised (a) 67 $\mathrm{Na}^{+}-23 \mathrm{Cl}^{-}$ions and $2.84 \times 10^{4}-3.03 \times 10^{4}$ $\mathrm{H}_{2} \mathrm{O}$ molecules averaged over a time period of $1.409 \mathrm{~ns}$; (b) $51 \mathrm{Na}^{+} 23 \mathrm{Cl}^{-}$ions and $1.38 \times$ $10^{4}-1.51 \times 10^{4} \mathrm{H}_{2} \mathrm{O}$ molecules averaged over a time period 1.851 ns.

In our simulations we choose as an initial condition a configuration taken from the equilibrium ensemble. The simulations have been performed by placing the droplet in vacuo and thermalizing it at $T=450 \mathrm{~K}$. In order to analyze the location of ions in a rapidly evaporating droplet the trajectory of the simulation was separated into blocks between ion evaporation events. In the blocks the number of $\mathrm{H}_{2} \mathrm{O}$ molecules and ions in the droplet does not

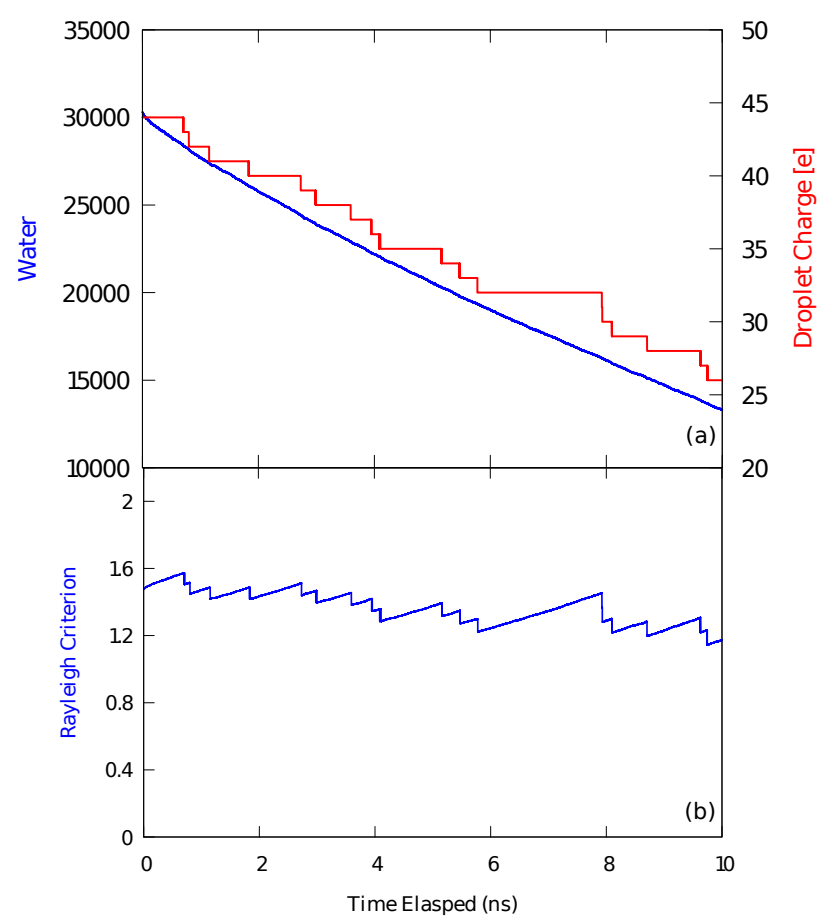

Figure 12: Evolution of an evaporating droplet composed of $3 \times 10^{4} \mathrm{H}_{2} \mathrm{O}$ molecules - $44 \mathrm{Na}^{+}$ ions - $23 \mathrm{NaCl}$ pairs at $T=450 \mathrm{~K}$. (a) Evolution of the number of $\mathrm{H}_{2} \mathrm{O}$ molecules and droplet the charge (b) Fissility parameter $(X)$ as a function of time.

change considerably. The radial ion distributions and the water denisty in representative blocks are shown in Fig. 11.

At $T=450 \mathrm{~K}$, the average water density in the interior of the water is $0.89 \mathrm{~g} / \mathrm{mL}$, which is significantly lower than the water density at room temperature. The width of the waterair interface is $\approx 3.0 \mathrm{~nm}$, which is significantly wider compared to $1.5 \mathrm{~nm}-1.7 \mathrm{~nm}$ at $T=350 \mathrm{~K}$. The distance between the water density and the ion concentration profile in the 10-90 interface gradually decreases as the droplet shrinks. This increase indicates that the rate of solvent evaporation is higher than the rate of ion diffusion.

Figure 12 (a) shows the evolution of droplet size and charge as a function of time and Fig. 12 (b) the time evolution of the fissility parameter. The trend is the same in droplets comprised $3 \times 10^{4}$ and $4 \times 10^{3} \mathrm{H}_{2} \mathrm{O}$ that we investigated (Fig. S7, S8 in SI). However, the smaller droplet may reach states with $X=1.2$ 
relative to the larger droplet that reaches states with $X=1.6$. Interestingly, the droplets with $\mathrm{Na}^{+}$and $\mathrm{Li}^{+}$ions show the same fragmentation pattern. These findings show that the droplets can be found transiently above the Rayleigh limit before they fragment. We note that IEM (by definition) should occur before the Rayleigh limit, therefore, IEM cannot be the mechanism that is followed when droplets are found above the Rayleigh limit.

Transient droplet states above the Rayleigh limit have been detected in experiments. Beauchamp et al. ${ }^{86}$ have reported that methanol microdrops with a small percentage of certain additive may fragment in a range of $112 \%-135 \%$ above the Rayleigh limit. This range corresponds to fissility parameter 1.251.825. Beauchamp et al. ${ }^{79,86}$ consider the temperature of the droplet to be that of the background gas, which is reported to be as high as $328 \mathrm{~K}$. This temperature is close to the boiling point of methanol, which is a quite elevated temperature for the droplets. Therefore, the conditions reported by Beauchamp et al. ${ }^{86}$ for methanol droplets likely cause faster solvent evaporation than ion diffusion to equilibrium positions. We propose that in addition to the temperature of the background gas, friction is another factor that may lead to an increase in the droplet temperature.

Now, we examine why droplets can transiently reach states above the Rayleigh limit. We propose two possible reasons that may delay the ion release. The first reason is that at the elevated temperature the Rayleigh limit may not hold because there is no surface tension since the droplets are found at a temperature above the solvent's boiling temperature. The $\mathrm{H}_{2} \mathrm{O}$ molecules are highly polarized in a thick outer droplet layer. This layer creates a cage for the ions that delays their release.

The second possible reason is the different time scales for conical formations and ion diffusion within the cones at elevated temperature. In the previous discussion, we examined the manner in which ions are emitted from the conical protrusions that appear on the droplet surface at $T=300 \mathrm{~K}-330 \mathrm{~K}$. This observation is also supported by other works that they have found experimentally and computationally that conical droplet deformations of a neutral droplet in an external electric field ${ }^{87}$ or via Rayleigh mechanism ${ }^{73,75}$ play a key role in the release of ions. In the high temperature, incipient cones rapidly undergo a death and birth process in different locations on the droplet surface. The ions do not diffuse fast enough to enter the cones. Therefore, the ion-release channel that dominates at lower temperatures cannot be followed at the elevated temperature.

Higashi et al. have found excellent agreement $^{80}$ of $\mathrm{MD}$ evaporation simulations at $T=$ $460 \mathrm{~K}$ with the IEM Labowsky et al. model. ${ }^{25}$ The simulated droplets comprise 2500 and 1000 water molecules with a mixture of $\mathrm{Na}^{+}$and $\mathrm{Cl}^{-}$ ions at a super-saturation. In light of our findings, the agreement between simulations and the Labowsky et al. model gives rise to a striking paradox. The main assumptions of the Labowsky et al. model are that (a) the free energy of activation arises from the Born ion solvation model and surface energy, which are equilibrium quantities, (b) all the ions are on the surface and (c) the charge of the ions is screened by the solvent, thus every solvated ion is not affected by the charge of the other ions. Under the conditions of the simulations reported by Higashi et al. (Ref. $\left[{ }^{80}\right]$ ) the assumptions of the Labowsky et al. model may not hold because (a) as we showed (Fig. 12) at $450 \mathrm{~K}$, when solvent evaporation is faster than the diffusion of ions toward the droplet interior, the ion distributions are not at equilibrium; (b) in solution of high ion concentration as that of the simulations of Higashi et al., ions share their solvation shell, thus, these solutions are characterized by low dielecric constant and the charge of the ions may not be screened by water in the smallest droplets of $1000 \mathrm{H}_{2} \mathrm{O}$ molecules; $30,88-94$ (c) here we showed that droplets at elevated temperature can be found above the Rayleigh limit, which excludes an IEM (in the IribarneThomson and Labowsky et al. meaning) by definition.

In summary we demonstrate that simulations under conditions where the solvent evaporation is faster than the ion diffusion can lead to transient droplet states above the Rayleigh limit. 
This finding may provide insight into experimental observations that find droplet states above the Rayleigh limit before fragmentation. Simulations of release of ions from minute nanodrops at elevated temperature cannot sufficiently establish the validity of an IEM (in the sense of Iribarne-Thomson and Labowsky et al.) and its agreement with the models.

\section{Conclusions}

The relation between the ion-ejection mechanisms and the composition of the surface excess charge layer in droplets was examined. We simulated the order of ejection of $\mathrm{Li}^{+}, \mathrm{Na}^{+}$and $\mathrm{Cs}^{+}$ions from droplets with a mixture of ions. The surface excess charge layer is enriched in $\mathrm{Li}^{+}$ions relative to $\mathrm{Na}^{+}$ions at the same concentration. In evaporation runs, at a temperature range of $300 \mathrm{~K}-340 \mathrm{~K} \mathrm{Li}^{+}$ions are ejected earlier than $\mathrm{Na}^{+}$ions. This finding is consistent with the equilibrium partition model of C. Enke. We observed that the higher the temperature the less the nature of the ion affects the order of ejection. A charged droplet close to the Rayleigh limit spontaneously develops conical protrusions. The cones are in general the locations from where the ions are ejected. Our finding supports the hypothesis that proteins and other macromolecules can be ejected from microdroplets by being captured in a conical deformation.

In the study of droplets with solvents of lower diectric constant than that of water we found that the lower the dielecric constant of a solvent, the wider the surface excess charge layer is. In a mixture of solvents, $\mathrm{CH}_{3} \mathrm{CN}-\mathrm{H}_{2} \mathrm{O}$ with $\mathrm{Na}^{+}$ions, the water is found in the droplet interior and contains the ions. The ions are released by the penetration of a water string within the $\mathrm{CH}_{3} \mathrm{CN}$ outer layer.

We found that the smaller the droplet, the smaller the $X$ value of a long-living metastable state. The charge states of the minute droplets may explain the experimentally observed charge state of small compact proteins such as ubiquitin, cytochrome $\mathrm{C}$, ovalbumin. ${ }^{82}$

Rapid evaporation of charged droplets consis- tently show that the systems can be transiently found above the Rayleigh limit before they fragment. We attribute the delay in the ion release to two factors (a) the use of the Rayleigh model is not justified at elevated temperature because the surface tension is not defined under these conditions (b) the short life-time of the conical deformations on the droplet surface relative to the diffusion time of the ions to the conical tips prevents the ions from following one of their ejection paths, which is prevalent at a lower temperature. This finding may provide an interpretation of the experiments ${ }^{79,86}$ that have detected droplets above the Rayleigh limit before their fission.

Minute droplet size or enrichment of the outer solvent layers in ions under rapid evaporation signals caution when simulations are used to validate models of the ion-evaporation mechanism. ${ }^{80}$ When evaporation is fast ions reside in non-equilibrium positions in the droplet's outer layer. Thus, their solvation does not obey the Born solvation model that underlies the ionevaporation models.

Direct evidence of IEM by atomistic modeling requires the following two conditions: (a) droplets of approximately $3 \times 10^{4} \mathrm{H}_{2} \mathrm{O}$ molecules and 50 ions. This system size is proposed because it may allow a jet of several ions to be released from conical deformations if a Rayleigh fission is followed. We support this proposal by the following scaling argument. Experimentally, it has been found that aqueous microdrops release 20\%-40\% of charge by Rayleigh mechanism. ${ }^{79}$ By scaling down this percentage to an aqueous droplet of $\approx 10^{3} \mathrm{H}_{2} \mathrm{O}$ molecules and 9-10 ions, it is found that 1-2 ions may be released. When 1-2 ions are ejected IEM and Rayleigh mechanism become indistinguishable. The larger droplet size that we propose may allow for differentiation from single-ion ejection. (b) For droplets that are subject to fragmentation dynamics temperature should be low enough so as the solvent evaporation is slower than the ion diffusion rate. We currently perform simulations in this direction.

Although in principle atomistic simulations appear to be the method that allows for the direct capture of the fission events, they may 
be misleading because the final residue of nonequilibrium molecular dynamics trajectories depends on the history of the droplet. We have argued in previous articles that droplets of diameter of at least $20 \mathrm{~nm}$ should be simulated in order to determine the solvation and charge states of macromolecules. ${ }^{95}$

In order to study the reactivity in electrosprayed droplets, it will be insightful to perform experiments in a mixture of solvents that can create a bilayer system, such as $\mathrm{H}_{2} \mathrm{O}$ and $\mathrm{CH}_{3} \mathrm{CN}$ in the presence of ions. This system may reveal the role of interfaces in altering reactivity.

The next question to address is how the presence of macromolecules may affect the preferential release of ions. Synergy between experiments and computations in exploring the effect of the mixture of ions in the charging of macromolecules and their ejection may provide insight into the charging mechanisms of macromolecules. We suggest $\mathrm{Li}^{+}$ion to be one of the candidate ions to use in experiments because its spatial distribution is clearly different from that of the other alkali metals and it also shares some commonalities with the hydronium ion such as its location in the outer droplet layers and quantum nuclear effects that play some role in its chemistry. The collected information will lead to a re-formulation of the ionevaporation mechanism.

The surface excess charge layer is the layer where chemical reactions that occur in the interface with vapor may couple to the droplet shape fluctuations. This coupling may play a more significant role in mesoscopic droplets. The composition of the surface excess charge layer also determines the type of ions that are ejected. Thus, this composition should be taken into account when examining release of small ionic species that are detected in mass spectrometry. The use of supervised and unsupervised machine learning has opened up new possibilities and interpretations in mass spectrometry imagining where a plethora of data are available. ${ }^{96-101}$ Similarly, we envision that a database of experimental and computational ion and macroion distributions in the surface excess charge layer may relate its composition to the mass spectrum.

\section{Appendix}

In all the simulations present and in previous research, we have found that the larger a droplet the closer to $X=1$ can exist for a longer period of time. The explanation of this behavior is as follows: The surface can be expressed as:

$$
\rho(\omega)=R+\sum_{l>0, m_{l}} a_{l, m_{l}} Y_{l, m_{l}}(\omega)
$$

where $\omega=(\theta, \phi)$ is the spherical angle, $\rho(\omega)$ is the distance from the centre, and $Y_{l m_{l}}(\omega)$ denote the spherical harmonics functions of rank $m$ and order $l$. $R$ is the $l=0$ term in the expansion of $\rho(\omega)$ and $a_{l m_{l}}$ are the amplitudes in the expansion of the surface fluctuations in terms of spherical harmonics. For certain shapes of droplets, such as bottle-necked shapes or "eight"-like shapes we should choose the center of the shape carefully, so as we do not have for a single $(\theta, \phi)$ more than one values of $\rho$. Equation 6 can also be written as

$$
\rho(\omega)=R\left[1+\sum_{l>0, m_{l}} \frac{a_{l, m_{l}}}{R} Y_{l, m_{l}}(\omega)\right]
$$

If we assume the same mechanism that leads to drop break-up for the smaller and larger drops, then $a_{l, m_{l}}$ is proportional to $R$. We make the above statement for the unstable modes, $l=2$ or in general for the modes that are important for droplet fragmentation. However this assumption may not be true for all the modes.

In a macroscopic description, the change in free energy of a droplet due to a perturbation from the spherical shape (but with fixed volume), can be expressed to lowest order as ${ }^{53,55}$

$$
\begin{gathered}
\delta E=\left(2 \pi R_{0}^{2} \gamma\right) \\
\sum_{l>0 \wedge|m| \leq l}(l-1)\left[(l+2)-\frac{Q^{2}}{(4 \pi)^{2} \varepsilon_{0} R_{0}^{3} \gamma}\right]\left|a_{l m_{l}}\right|^{2}
\end{gathered}
$$

where $Q, R_{0}$, and $\gamma$ denote the total charge of the droplet, the unperturbed droplet radius, 
and the surface tension, respectively, and $\varepsilon_{0}$ is the permittivity of vacuum.

Equation 8 can also be written as

$$
\begin{aligned}
\delta E & =\left(2 \pi R_{0}^{2} \gamma\right) \sum_{l>0 \wedge\left|m_{l}\right| \leq l}(l-1)[(l+2)-4 X]\left|a_{l m}\right|^{2} \\
& =\left(2 \pi R_{0}^{2} \gamma\right) \sum_{l>0 \wedge\left|m_{l}\right| \leq l} \eta_{l m_{l}}\left|a_{l m_{l}}\right|^{2}
\end{aligned}
$$

where $X$ is the fissility parameter and $\eta_{l m_{l}}=$ $(l-1)[(l+2)-4 X]$.

The charged droplets are metastable states even if they are much below the Rayleigh limit. We compare two droplets with radii $R^{\prime}$ and $R^{\prime \prime}$. The rate constant is given by

$$
k(R) \sim \exp \left(-E^{*} / k_{B} T\right)
$$

We take the rate of fragmentation to be the same in droplets of different size, thus,

$$
k\left(R^{\prime}\right) \sim k\left(R^{\prime \prime}\right) \Rightarrow \eta_{l m}\left(R^{\prime}\right)\left|a_{l m}^{\prime}\right|^{2} \sim \eta_{l m}\left(R^{\prime \prime}\right)\left|a_{l m}^{\prime \prime}\right|^{2}
$$

If the same mechanism of break-up holds, i.e. the same fluctuations appear in the barrier top, then, $a_{l, m_{l}} / R$ is a constant. Then, Eq. 13 for $l=2$ becomes

$$
\eta_{l m}\left(R^{\prime}\right) R^{\prime 2} \sim \eta_{l m}\left(R^{\prime \prime}\right) R^{\prime \prime 2} \Rightarrow \frac{1-X^{\prime}}{1-X^{\prime \prime}} \sim\left(\frac{R^{\prime \prime}}{R^{\prime}}\right)^{2}
$$

Therefore, the smaller the droplet the smaller the $X$ for fragmentation.

Acknowledgement S.C. greatly thanks Prof. D. Frenkel, Department of Chemistry, University of Cambridge, UK, Professor Raymond Kapral, Department of Chemistry, University of Toronto and Dr. Anatoly Malevanets and Professor C. Enke, The University of New Mexico, for discussions on the stability of charged systems. Belated thankfulness is expressed to Prof. Dr. Ron Heeren, Maastricht MultiModal Molecular Imaging Institute and Prof. Daniel Laria, Department of Inorganic Chemistry, University of Buenos Aires who enthusiastically supported and discussed the computational research of the highly charged droplets at its infancy in 1997-1999 in the Department of Chemistry of the University of Toronto and in AMOLF, Amsterdam, The Netherlands, respectively. S.C. acknowledges an NSERC-Discovery grant (Canada) for funding this research. V.K. acknowledges the province of Ontario and the University of Western Ontario for the Queen Elizabeth II Graduate Scholarship in Science and Technology. J.T. acknowledges a MITACS Globalink Research Internship in the Consta group. ComputeCanada is acknowledged for providing the computing facilities.

\section{References}

(1) Shi, Z.; Tan, Y.; Tang, H.; Sun, J.; Yang, Y.; Peng, L.; Guo, X. Aerosol effect on the land-ocean contrast in thunderstorm electrification and lightning frequency. Atmos. Res. 2015, 164, 131-141.

(2) Yudistira, H. T.; Nguyen, V. D.; Dutta, P.; Byun, D. Flight behavior of charged droplets in electrohydrodynamic inkjet printing. Appl. Phys. Lett. 2010, 96, 023503.

(3) Loo, R. R. O.; Lakshmanan, R.; Loo, J. A. What protein charging (and supercharging) reveal about the mechanism of electrospray ionization. J. Am. Soc. Mass. Spectrom. 2014, 25, 16751693.

(4) Mehmood, S.; Allison, T. M.; Robinson, C. V. Mass spectrometry of protein complexes: From origins to applications. Annu. Rev. Phys. Chem. 2015, 66, 453474.

(5) Hirabayashi, A.; Sakairi, M.; Koizumi, H. Sonic spray ionization method for atmospheric pressure ionization mass spectrometry. Anal. Chem. 1994, 66, 45574559.

(6) Blakley, C.; Vestal, M. Thermospray interface for liquid chromatography/mass spectrometry. Anal. Chem. 1983, 55, 750-754. 
(7) Monge, M. E.; Harris, G. A.; Dwivedi, P.; Fernandez, F. M. Mass spectrometry: recent advances in direct open air surface sampling/ionization. Chem. Rev. 2013, 113, 2269-2308.

(8) Devereaux, Z. J.; Reynolds, C. A.; Fischer, J. L.; Foley, C. D.; DeLeeuw, J. L.; Wager-Miller, J.; Narayan, S. B.; Mackie, K.; Trimpin, S. Matrix-Assisted Ionization on a Portable Mass Spectrometer: Analysis Directly from Biological and Synthetic Materials. Anal. Chem. 2016, 88, 10831-10836.

(9) Sakairi, M.; Kambara, H. Atmospheric pressure spray ionization for liquid chromatography/mass spectrometry. Anal. Chem. 1989, 61, 1159-1164.

(10) Bruins, A. P.; Covey, T. R.; Henion, J. D. Ion spray interface for combined liquid chromatography/atmospheric pressure ionization mass spectrometry. Anal. Chem. 1987, 59, 2642-2646.

(11) Kambara, H. Sample introduction system for atmospheric pressure ionization mass spectrometry of nonvolatile compounds. Anal. Chem. 1982, 54, 143-146.

(12) Bain, R. M.; Pulliam, C. J.; Cooks, R. G. Accelerated Hantzsch electrospray synthesis with temporal control of reaction intermediates. Chem. Sci. 2015, 6, 397401.

(13) Chen, X.; Cooks, R. G. Accelerated reactions in field desorption mass spectrometry. J. Mass Spectrom. 2018, 53, 942946.

(14) Schrader, R. L.; Fedick, P. W.; Mehari, T. F.; Cooks, R. G. Accelerated Chemical Synthesis: Three Ways of Performing the Katritzky Transamination Reaction. J. Chem. Educ. 2019,

(15) Lee, J. K.; Banerjee, S.; Nam, H. G.; Zare, R. N. Acceleration of reaction in charged microdroplets. Q. Rev. Biophys. 2015, 48, 437-444.

(16) Ingram, A. J.; Boeser, C. L.; Zare, R. N. Going beyond electrospray: mass spectrometric studies of chemical reactions in and on liquids. Chem. Sci. 2016, 7, 3955 .

(17) Sahraeian, T.; Kulyk, D. S.; BaduTawiah, A. K. Droplet Imbibition Enables Non-Equilibrium Interfacial Reactions in Charged Microdroplets. Langmuir 2019, 35, 14451-14457.

(18) Kulyk, D. S.; Miller, C. F.; BaduTawiah, A. K. Reactive charged droplets for reduction of matrix effects in electrospray ionization mass spectrometry. Anal. Chem. 2015, 87, 10988-10994.

(19) Malik, S. A.; Ng, W. H.; Bowen, J.; Tang, J.; Gomez, A.; Kenyon, A. J.; Day, R. M. Electrospray synthesis and properties of hierarchically structured PLGA TIPS microspheres for use as controlled release technologies. J. Colloid Interface Sci. 2016, 467, 220-229.

(20) de la Mora, J. F. Electrospray ionization of large multiply charged species proceeds via Dole's charged residue mechanism. Anal. Chim. Acta 2000, 406, 93104.

(21) Wilm, M. Principles of electrospray ionization. Mol. Cell. Proteomics 2011, 10, M111-009407.

(22) Kebarle, P.; Verkerk, U. H. Electrospray: from ions in solution to ions in the gas phase, what we know now. Mass Spectrom. Rev. 2009, 28, 898-917.

(23) Iribarne, J. V.; Thomson, B. A. On the evaporation of small ions from charged droplets. J. Chem. Phys. 1976, 64, 22872294.

(24) Thomson, B.; Iribarne, J. Field induced ion evaporation from liquid surfaces at atmospheric pressure. J. Chem. Phys. 1979, 71, 4451-4463. 
(25) Labowsky, M.; Fenn, J.; de la Mora, J. F. A continuum model for ion evaporation from a drop: effect of curvature and charge on ion solvation energy. Anal. Chim. 2000, 406, 105-118.

(26) Hogan Jr, C. J.; Carroll, J. A.; Rohrs, H. W.; Biswas, P.; Gross, M. L. Combined charged residue-field emission model of macromolecular electrospray ionization. Anal. Chem. 2008, 81, 369377.

(27) Consta, S.; Mainer, K. R.; Novak, W. Fragmentation mechanisms of aqueous clusters charged with ions. J. Chem. Phys. 2003, 119, 10125-10132.

(28) Kwan, V.; Malevanets, A.; Consta, S. Where do the ions reside in a highly charged droplet? J. Phys. Chem. A 2019, 123, 9298-9310, PMID: 31589448.

(29) Kwan, V.; Consta, S. Bridging electrostatic properties between nanoscopic and microscopic highly charged droplets. Chem. Phys. Lett. 2020, $746,137238$.

(30) Kwan, V.; Consta, S. Molecular Characterization of the Surface Excess Charge Layer in Droplets. J. Am. Soc. Mass Spectrom. 2021, 32, 33-45.

(31) Dole, M.; Mack, L.; Hines, R.; Mobley, R.; Ferguson, L.; Alice, M. d. Molecular beams of macroions. J. Chem. Phys. 1968, 49, 2240-2249.

(32) Consta, S. Manifestation of Rayleigh instability in droplets containing multiply charged macroions. J. Phys. Chem. B 2010, 114, 5263-5268.

(33) Consta, S.; Oh, M. I.; Malevanets, A. New mechanisms of macroion-induced disintegration of charged droplets. Chem. Phys. Lett. 2016, 663, 1-12.

(34) Consta, S.; Malevanets, A. Classification of the ejection mechanisms of charged macromolecules from liquid droplets. $J$. Chem. Phys. 2013, 138, 044314.
(35) Malevanets, A.; Consta, S. Variation of droplet acidity during evaporation. J. Chem. Phys. 2013, 138, 184312.

(36) Oh, M. I.; Consta, S. Charging and Release Mechanisms of Flexible Macromolecules in Droplets. J. Am. Soc. Mass. Spectrom. 2017, 28, 2262-2279.

(37) Consta, S.; Chung, J. K. Chargeinduced conformational changes of PEG$(\mathrm{Na}(\mathrm{n})(+))$ in a vacuum and aqueous nanodroplets. J. Phys. Chem. B 2011, 115, 10447-10455.

(38) Rayleigh, L. XX. On the equilibrium of liquid conducting masses charged with electricity. Philos. Mag. 1882, 14, 184186.

(39) Labowsky, M. Discrete charge distributions in dielectric droplets. J. Colloid Interface Sci. 1998, 206, 19-28.

(40) Colussi, A. J.; Enami, S. Detecting intermediates and products of fast heterogeneous reactions on liquid surfaces via online mass spectrometry. Atmosphere 2019, 10, 47.

(41) Enami, S.; Sakamoto, Y.; Colussi, A. J. Fenton chemistry at aqueous interfaces. Proc. Natl. Acad. Sci. U.S.A. 2014, 111, 623-628.

(42) Colussi, A. J. Can the pH at the air/water interface be different from the pH of bulk water? Proc. Natl. Acad. Sci. USA 2018, 115, E7887.

(43) Enami, S.; Hoffmann, M. R.; Colussi, A. J. Proton availability at the air/water interface. J. Phys. Chem. Lett. 2010, 1, 1599-1604.

(44) Mishra, H.; Enami, S.; Nielsen, R. J.; Stewart, L. A.; Hoffmann, M. R.; Goddard, W. A.; Colussi, A. J. Brønsted basicity of the air-water interface. Proc. Natl. Acad. Sci. U.S.A. 2012, 109, 18679-18683. 
(45) Agmon, N.; Bakker, H. J.; Campen, R. K.; Henchman, R. H.; Pohl, P.; Roke, S.; Thämer, M.; Hassanali, A. Protons and hydroxide ions in aqueous systems. Chem. Rev. 2016, $116,7642-7672$.

(46) Narendra, N.; Chen, X.; Wang, J.; Charles, J.; Cooks, R. G.; Kubis, T. Quantum Mechanical Modeling of Reaction Rate Acceleration in Microdroplets. J. Chem. Phys. A 2020, 124, 4984-4989.

(47) Buch, V.; Milet, A.; Vácha, R.; Jungwirth, P.; Devlin, J. P. Water surface is acidic. Proc. Natl. Acad. Sci. U.S.A. 2007, 104, 7342-7347.

(48) Roux, B.; Yu, H. A.; Karplus, M. Molecular basis for the Born model of ion solvation. J. Phys. Chem. 1990, 94, 46834688 .

(49) Enke, C. G. A predictive model for matrix and analyte effects in electrospray ionization of singly-charged ionic analytes. Anal. Chem. 1997, 69, 4885-4893.

(50) Constantopoulos, T. L.; Jackson, G. S.; Enke, C. G. Challenges in achieving a fundamental model for ESI. Anal. Chim. Acta 2000, 406, 37-52.

(51) Hogan Jr, C. J.; Carroll, J. A.; Rohrs, H. W.; Biswas, P.; Gross, M. L. Charge carrier field emission determines the number of charges on native state proteins in electrospray ionization. $J$. Am. Chem. Soc. 2008, 130, 6926-6927.

(52) Chamberlayne, C. F.; Zare, R. N. Simple model for the electric field and spatial distribution of ions in a microdroplet. $J$. Chem. Phys. 2020, 152, 184702.

(53) Hendricks, C.; Schneider, J. Stability of a conducting droplet under the influence of surface tension and electrostatic forces. Am. J. Phys 1963, 31, 450-453.

(54) Peters, J. Rayleigh's electrified water drops. Eur. J. Phys. 1980, 1, 143.
(55) Consta, S.; Malevanets, A. Disintegration mechanisms of charged nanodroplets: novel systems for applying methods of activated processes. Molec. Simul. 2015, 41, 73-85.

(56) Oh, M. I.; Malevanets, A.; Paliy, M.; Frenkel, D.; Consta, S. When droplets become stars: charged dielectric droplets beyond the Rayleigh limit. Soft Matter 2017, 13, 8781-8795.

(57) Phillips, J. C.; Braun, R.; Wang, W.; Gumbart, J.; Tajkhorshid, E.; Villa, E.; Chipot, C.; Skeel, R. D.; Kalé, L.; Schulten, K. Scalable molecular dynamics with NAMD. J. Comput. Chem. 2005, 26, 1781-1802.

(58) Humphrey, W.; Dalke, A.; Schulten, K. VMD: Visual Molecular Dynamics. $J$. Mol. Graphics 1996, 14, 33-38.

(59) Jorgensen, W. L.; Jenson, C. Temperature dependence of TIP3P, SPC, and TIP4P water from NPT Monte Carlo simulations: Seeking temperatures of maximum density. J. Comput. Chem. 1998, 19, 1179-1186.

(60) Noskov, S. Y.; Roux, B. Control of Ion Selectivity in LeuT: Two Na+ Binding Sites with Two Different Mechanisms. J. Mol. Biol. 2008, 37r, $804-818$.

(61) Beglov, D.; Roux, B. Finite representation of an infinite bulk system: Solvent boundary potential for computer simulations. J. Chem. Phys. 1994, 100, 90509063.

(62) Vanommeslaeghe, K.; Hatcher, E.; Acharya, C.; Kundu, S.; Zhong, S.; Shim, J.; Darian, E.; Guvench, O.; Lopes, P.; Vorobyov, I. et al. CHARMM general force field: A force field for drug-like molecules compatible with the CHARMM all-atom additive biological force fields. J. Comp. Chem. 2010, 31, 671-690. 
(63) Vega, C.; de Miguel, E. Surface tension of the most popular models of water by using the test-area simulation method. $J$. Chem. Phys. 2007, 126, 154707.

(64) Hardy, D. J.; Wu, Z.; Phillips, J. C.; Stone, J. E.; Skeel, R. D.; Schulten, K. Multilevel summation method for electrostatic force evaluation. J. Chem. Theory Comput. 2015, 11, 766-779.

(65) Lyubartsev, A.; Laasonen, K.; Laaksonen, A. d. Hydration of $\mathrm{Li}+$ ion. An ab initio molecular dynamics simulation. $J$. Chem. Phys. 2001, 114, 3120-3126.

(66) Pye, C. C.; Rudolph, W.; Poirier, R. A. An ab initio investigation of lithium ion hydration. J. Phys. Chem. 1996, 100, 601-605.

(67) Rudolph, W.; Brooker, M. H.; Pye, C. C. Hydration of lithium ion in aqueous solutions. J. Phys. Chem. 1995, 99, 37933797 .

(68) Loeffler, H. H.; Rode, B. M. The hydration structure of the lithium ion. $J$. Chem. Phys. 2002, 117, 110-117.

(69) Newsome, J.; Neilson, G.; Enderby, J. Lithium ions in aqueous solution. $J$. Phys. C 1980, 13, L923.

(70) Matsuda, Y.; Fukushima, T.; Hashimoto, H.; Arakawa, R. Solvation of lithium ions in mixed organic electrolyte solutions by electrospray ionization mass spectroscopy. J. Electrochem. Soc. 2002, 149, A1045.

(71) Lekner, J.; Henderson, J. Theoretical determination of the thickness of a liquidvapour interface. Physica A: Statistical Mechanics and its Applications 1978, 94, 545-558.

(72) Zakharov, V. V.; Brodskaya, E. N.; Laaksonen, A. Surface tension of water droplets: A molecular dynamics study of model and size dependencies. J. Chem. Phys. 1997, 10\%, 10675-10683.
(73) Gomez, A.; Tang, K. Charge and fission of droplets in electrostatic sprays. Phys. Fluids 1994, 6, 404-414.

(74) Duft, D.; Lebius, H.; Huber, B. A.; Guet, C.; Leisner, T. Shape Oscillations and Stability of Charged Microdroplets. Phys. Rev. Lett. 2002, 89, 084503.

(75) Duft, D.; Achtzehn, T.; Muller, R.; Huber, B. A.; Leisner, T. Coulomb fission: Rayleigh jets from levitated microdroplets. Nature 2003, 421, 128-128.

(76) Taylor, G. Disintegration of Water Drops in an Electric Field. Proceedings of the Royal Society of London A: Mathematical, Physical and Engineering Sciences 1964, 280, 383-397.

(77) R Core Team, R: A Language and Environment for Statistical Computing. $\mathrm{R}$ Foundation for Statistical Computing: Vienna, Austria, 2017.

(78) Consta, S. Fragmentation reactions of charged aqueous clusters. J. Mol. Struct. THEOCHEM 2002, 591, 131-140.

(79) Smith, J. N.; Flagan, R. C.; Beauchamp, J. Droplet evaporation and discharge dynamics in electrospray ionization. J. Phys. Chem. A 2002, 106, 9957-9967.

(80) Higashi, H.; Tokumi, T.; Hogan, C. J.; Suda, H.; Seto, T.; Otani, Y. Simultaneous ion and neutral evaporation in aqueous nanodrops: experiment, theory, and molecular dynamics simulations. Phys. Chem. Chem. Phys. 2015, 17, 1574615755 .

(81) Ahadi, E.; Konermann, L. Ejection of solvated ions from electrosprayed methanol/water nanodroplets studied by molecular dynamics simulations. J. Am. Chem. Soc. 2011, 133, 9354-9363.

(82) Hogan Jr, C. J.; Carroll, J. A.; Rohrs, H. W.; Biswas, P.; Gross, M. L. Combined charged residue-field emission 
model of macromolecular electrospray ionization. Anal. Chem. 2009, 81, 369377.

(83) Rovelli, G.; Jacobs, M. I.; Willis, M. D.; Rapf, R. J.; Prophet, A. M.; Wilson, K. R. A critical analysis of electrospray techniques for the determination of accelerated rates and mechanisms of chemical reactions in droplets. Chem. Sci. 2020, 11, 13026-13043.

(84) Soleilhac, A.; Dagany, X.; Dugourd, P.; Girod, M.; Antoine, R. Correlating droplet size with temperature changes in electrospray source by optical methods. Anal. Chem. 2015, 87, 8210-8217.

(85) Gibson, S. C.; Feigerle, C. S.; Cook, K. D. Fluorometric measurement and modeling of droplet temperature changes in an electrospray plume. Anal. Chem. 2013, $86,464-472$.

(86) Grimm, R. L.; Beauchamp, J. Evaporation and discharge dynamics of highly charged multicomponent droplets generated by electrospray ionization. J. Phys. Chem. A 2009, 114, 1411-1419.

(87) Luedtke, W. D.; Landman, U.; Chiu, Y.H.; Levandier, D. J.; Dressler, R. A.; Sok, S.; Gordon, M. S. Nanojets, Electrospray, and Ion Field Evaporation: Molecular Dynamics Simulations and Laboratory Experiments. J. Phys. Chem. A 2008, 112, 9628-9649.

(88) Gavish, N.; Promislow, K. Dependence of the dielectric constant of electrolyte solutions on ionic concentration: A microfield approach. Phys. Rev. E 2016, 94, 012611.

(89) Hyun, J.-K.; Babu, C. S.; Ichiye, T. Apparent local dielectric response around ions in water: a method for its determination and its applications. J. Phys. Chem. 1995, 99, 5187-5195.
(90) Haggis, G.; Hasted, J.; Buchanan, T. The dielectric properties of water in solutions. J. Chem. Phys. 1952, 20, 1452-1465.

(91) Chandra, A. Static dielectric constant of aqueous electrolyte solutions: Is there any dynamic contribution? J. Chem. Phys. 2000, 113, 903-905.

(92) Friedman, H. Theory of the dielectric constant of solutions. J. Chem. Phys. 1982, 76, 1092-1105.

(93) Chan, D. Y.; Mitchell, D. J.; Ninham, B. W. A model of solvent structure around ions. J. Chem. Phys. 1979, 70, 2946-2957.

(94) Levy, A.; Andelman, D.; Orland, H. Dielectric constant of ionic solutions: A field-theory approach. Phys. Rev. Lett. 2012, 108, 227801.

(95) Consta, S.; Oh, M. I.; Kwan, V.; Malevanets, A. Strengths and Weaknesses of Molecular Simulations of Electrosprayed Droplets. J. Am. Soc. Mass Spectrom. 2018, 29, 2287-2296.

(96) Allen, F.; Pon, A.; Greiner, R.; Wishart, D. Computational prediction of electron ionization mass spectra to assist in GC/MS compound identification. Anal. Chem. 2016, 88, 7689-7697.

(97) Hua, D.; Liu, X.; Go, E. P.; Wang, Y.; Hummon, A. B.; Desaire, H. How to Apply Supervised Machine Learning Tools to MS Imaging Files: Case Study with Cancer Spheroids Undergoing Treatment with the Monoclonal Antibody Cetuximab. J. Am. Soc. Mass Spectrom. 2020, 31, 1350-1357.

(98) Swan, A. L.; Mobasheri, A.; Allaway, D.; Liddell, S.; Bacardit, J. Application of machine learning to proteomics data: classification and biomarker identification in postgenomics biology. OMICS 2013, 17, 595-610. 
(99) Verbeeck, N.; Caprioli, R. M.; Van de Plas, R. Unsupervised machine learning for exploratory data analysis in imaging mass spectrometry. Mass Spectrom. Rev. 2020, 39, 245-291.

(100) Liebal, U. W.; Phan, A. N.; Sudhakar, M.; Raman, K.; Blank, L. M. Machine learning applications for mass spectrometry-based metabolomics. Metabolites 2020, 10, 243.

(101) Wei, J. N.; Belanger, D.; Adams, R. P.; Sculley, D. Rapid prediction of electronionization mass spectrometry using neural networks. ACS Cent. Sci. 2019, 5, 700-708. 